\title{
PROPERTIES OF COMPLEX CHAOS IN CONDITIONAL QUBIT DYNAMICS
}

\author{
T. KISS \\ Research Institute for Solid State Physics and Optics, \\ H-1525 Budapest, P. O. Box 49, Hungary \\ tkiss@szfki.hu \\ I. JEX \\ Department of Physics, FJFI ČVUT, \\ Břehová 7, 11519 Praha 1 - Staré Město, Czech Republic \\ G. ALBER \\ Institut für Angewandte Physik, Technische Universität Darmstadt, \\ D-64289 Darmstadt, Germany \\ E. KOLLÁR \\ Eötvös University, Pázmány P. stny. 1 A, \\ H-1117 Budapest, Hungary
}

Received 27 February 2008

\begin{abstract}
Complex chaos is specified by an iterated mapping on complex numbers. It has recently been found in the dynamics of qubits where each time step is conditioned on a measurement result on part of the system. We analyse the simplest case of one qubit dynamics with one complex parameter in some detail. We point out that two attractive cycles can exist and provide examples how the fractal like Julia set divides the areas of corresponding initial states. We show how to determine the set of parameters for which one, two or no stable fixed cycles exists and provide the numerically calculated images of the sets. The results can be relevant for the quantum state purification protocol based on the similar dynamics of two or more qubits and in general for any protocol based on conditioned nonlinear dynamics where truly chaotic behavior may occur.
\end{abstract}

Keywords: Quantum chaos; complex chaos; purification.

\section{Introduction}

Quantum dynamics is described by completely positive maps. From the physical point of view, two types of maps are clearly distinguished. On one hand isolated, closed systems evolve unitarily, i.e. the map consists of the action of a unitary operator. On the other hand interaction with other systems and especially measurement changes the character of the evolution and in general it will not be unitary for open systems. Unitarity of the evolution excludes exponential sensitivity with respect to 
initial conditions, thus true chaos cannot exist in a closed quantum system. This strong statement is strictly valid for the distance of two quantum states in the Hilbert space. Note that in a more restricted sense, considering distance only in the configuration space, one can find chaotic quantum systems. ${ }^{1}$ We note here that the term quantum chaos usually refers to the studies of the unitary evolution of quantum systems with classically chaotic counterparts. ${ }^{2}$

Measurements on a system break unitarity, and it has been proven that the quantized version of a classically chaotic system can be chaotic in the quantum domain as well if it is continuously measured. ${ }^{3}$ Another proposal suggests to feed back the results of weak measurements ${ }^{4}$ in order to introduce nonlinearity which probably could lead to truly chaotic behavior (however, we are not aware of a proof of the latter statement).

A rather different way of considering measurements emerges in the context of quantum information theory. Here it is natural to apply measurements on an ensemble of systems in order to select a subensemble according to the measurement result. For example, purification protocols are based on such a procedure. In this way nonlinearity can be introduced into an originally linear system. ${ }^{5}$ In turn, the nonlinear, conditional dynamics of qubits can lead to true chaos, with no trivial classical counterpart. ${ }^{6}$ The underlying map is a complex to complex function, with one complex parameter, therefore the name complex chaos has been coined.

In this paper we present results on the complex, truly chaotic dynamics of qubits, showing that at most two distinct stable fixed cycles may exist when the system in consideration consists of a single qubit. The corresponding areas in the parameter space are determined numerically.

\section{Iterated Nonlinear Dynamics of Qubits}

The purification scheme we analyze relies on the availability of an ensemble of identically prepared $n$-qudit systems. Let us consider these systems pairwise and apply a quantum XOR gate on each pair, where

$$
X O R_{12}|i\rangle_{1}|j\rangle_{2}=|i\rangle_{1}|i \ominus j\rangle_{2}
$$

Here $D$ is the dimension of the Hilbert space for each system, the symbol $\ominus$ stands for difference modulo $D$ and $i, j$ label basis elements in the $D$ dimensional Hilbert space. After the application of the quantum XOR gate let us make a measurement on the second system in the computational basis and keep the other member of the pair only if the result was zero. This procedure selects subensemble of systems in an identical state. The procedure can be repeated starting from the subensemble. Thus by iteration one arrives at a highly nonlinear dynamics. The scheme can be used for quantum state purification ${ }^{5}$ which is especially useful if the systems consist of at least two qubits $(D=4)$.

The single qubit case $(D=2)$ is somewhat simpler, but still leads to rich dynamics. We introduce the following notation for the description of the qubit in a 
pure state

$$
|\psi\rangle=N(z|0\rangle+|1\rangle)
$$

where the normalization reads $N=\left(1+|z|^{2}\right)^{-1 / 2}$. Each step of the iterated evolution consists of the nonlinear transformation and an arbitrary but fixed unitary operation on the qubit

$$
U=\left(\begin{array}{cc}
\cos x & \sin x e^{i \phi} \\
-\sin x e^{-i \phi} & \cos x
\end{array}\right),
$$

in the prescribed basis. An initial pure state is always mapped onto a pure state, therefore a complex to complex function describes each step of the evolution

$$
z \mapsto F_{p}(z)=\frac{z^{2}+p}{1-p^{*} z^{2}},
$$

where $p=\tan x e^{i \phi}$ and the star denotes complex conjugation. In the next section we discuss some properties of dynamics evoked by (4), which is a rational function of degree two.

\section{Properties of the Single Qubit Dynamics}

The iterated complex map (4) has two critical points: $z_{c 1}=0$ and $z_{c 2}=\infty$ where its derivative vanishes (for $\infty$ the reciprocal of the limit of the derivative must be taken). Fixed cycles of the map are defined by $F_{p}^{\circ n}\left(z_{0}\right)=z_{0}$. The absolute value of the multiplier of the periodic orbit, $\Lambda=\left(F_{p}^{\circ n}\right)^{\prime}\left(z_{0}\right)$ determines its stability. For $|\Lambda|<1,|\Lambda|>1,|\Lambda|=1$ the cycles are attractive, repelling and neutral, respectively. An important theorem on dynamical maps ${ }^{7}$ ensures that for a fixed parameter value $p$ by following the orbit of the critical points one can find all attractive fixed cycles. Consequently, the map (4) can have at most two attractive cycles.

The complex plane of the initial values $z_{0}$ can be divided into regular and irregular points forming the Fatou and Julia sets, respectively. Regular starting points from the Fatou set will converge to a stable cycle (also elements of the Fatou set) when repeating the iteration. Initial values included in the Julia set are considered to be chaotic, leading to irregular oscillations or forming unstable cycles. In Fig. 1. details of the Julia and Fatou sets are shown for a given parameter value. One can observe self-similar patterns in the Julia set, which is a common property for Julia sets of rational maps, related to the fractal structure of the set. The Julia set becomes a circle ${ }^{6}$ for $p=0$, for which case a generalized Lyapunov exponent can be calculated with the value $\lambda_{0}=2 \ln 2$.

In the case of two different attractive periodic orbits the Julia set separates the two regions of convergence, i.e. convergence to one or to the other stable cycle. Such a situation is demonstrated in Fig. 2. The fractal structure of the Julia set implies that around certain points changing the initial state on any small scale may alter the convergence of the evolution from one fixed cycle to the other or no convergence 
Julia set for $\mathrm{p}=0.05+0.6 \mathrm{i}$

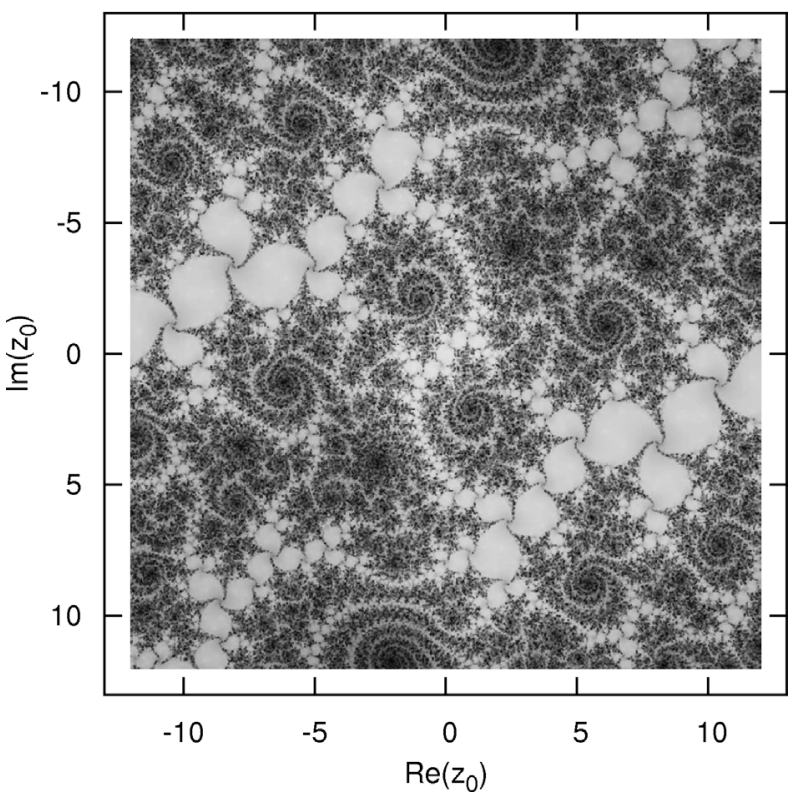

Fig. 1. Julia set of initial states with 1 fixed cycle. Gray scale indicates the speed of convergence to the attractive cycle $\left\{z_{1} \approx 0.06+0.58 i, z_{2} \approx-0.41+0.6 i, z_{3} \approx-0.11+0.07 i\right\}$. Light gray: fast convergence, dark gray: slow convergence, black: Julia set. The parameter value is set to $p=0.05+0.6 i$.

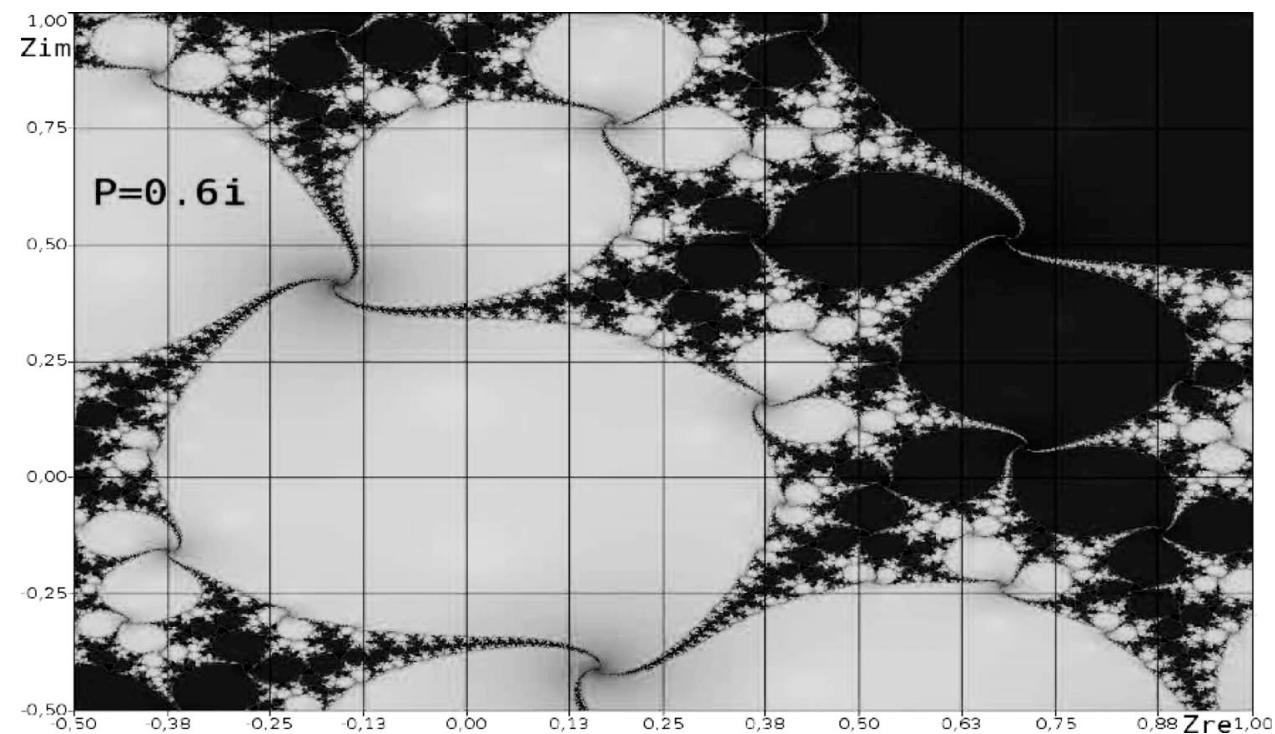

Fig. 2. Julia set of initial states with 2 attractive fixed cycles. The parameter value is set to $p=0.6 i$. Light and dark gray indicate convergence to the two stable fixed cycles, respectively. The Julia set occupies the border of the two regions (black). 


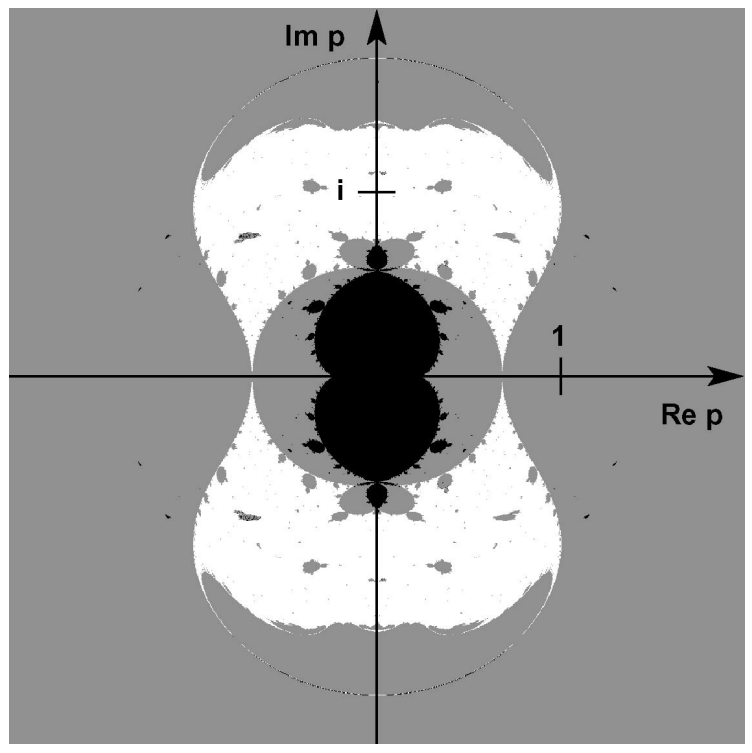

Fig. 3. The number of attractive periodic orbits in the parameter space. Black indicates the existence of two distinct stable cycles, gray means a single stable cycle and white is the fully chaotic region where no attractive periodic orbits exist. The image was generated by numerically following the orbit of the two critical points and testing for convergence.

at all. Note that a small change of the parameter value results in a change of the character of the dynamics.

The number of stable cycles depends on the parameter of the dynamics. By following the orbit of the critical points and testing for convergence, one can determine the number of distinct stable cycles. An overview is provided by the parameter space picture in Fig. 3, where the regions with two, one or no attractive periods are shown. Shapes resembling those of the the Mandelbrot set are formed suggesting self similarity and fine structures on any small scale.

\section{Conclusion}

Our results on complex dynamics of qubits can have interesting implications for the currently discussed feed forward schemes. They indicate that feeding forward the measurement results can in certain cases lead to a destabilization (chaos) in contrast to the expected stabilization of the dynamics.

The procedure of feed-forward generates nonlinearity which affects purification protocols. ${ }^{5}$ Emerging complex chaos results in a delicate behavior, sensitive to the initial state as well as the parameter of the dynamics. The fractal nature of the Julia set of initial states means that changes on any scale in the initial condition can have a dramatic effect by changing the stability of the fixed cycle. The one qubit system provides a toy model for conditional, iterative dynamics exhibiting all 
the above mentioned features. Numerical results indicate a behavior very similar to the single qubit case also to the case of purifying entangled qubits.

\section{Acknowledgment}

The financial support by MSM 6840770039, MŠMT LC 06002, the Czech-Hungarian cooperation project (KONTAKT,CZ-2/2005), by the Hungarian Scientific Research Fund (T049234) and by DAAD is acknowledged.

\section{References}

1. S. Weigert, Phys. Rev. A 48 (1993) 1780.

2. M.-J. Giannoni, A. Voros and J. Zinn-Justin, Chaos and Quantum Physics, Proceedings of the Les Houches Lecture Series, Session 52 (North-Holland, Amsterdam, 1991); P. Cvitanović, R. Artuso, R. Mainieri, G. Tanner and G. Vattay, Chaos: Classical and Quantum (Niels Bohr Institute, Copenhagen, 2005), ChaosBook.org.

3. S. Habib, K. Jacobs and K. Shizume, Phys. Rev. Lett. 96 (2006) 010403; S. Habib et al., e-print quant-ph/0505085.

4. S. Lloyd and J.-J. Slotine Phys. Rev. A 62 (2000) 012307.

5. H. Bechmann-Pasquinucci et al., Phys. Lett. A 242 (1998) 198; D. R. Terno, Phys. Rev. A 59 (1999) 3320; G. Alber et al., J. Phys. A: Math. Gen. 34 (2001) 8821.

6. T. Kiss, I. Jex, G. Alber and S. Vymetal, Phys. Rev. A 74 (2006) 040301R; Acta Phys. Hung. B 26 (2006) 229.

7. J. W. Milnor, Dynamics in One Complex Variable (Vieweg, 2000). 